This is an electronic reprint of the original article. This reprint may differ from the original in pagination and typographic detail.

\author{
Author(s): Joffre, Thomas; Miettinen, Arttu; Wernersson, Erik L.G.; Isaksson, Per; Gamstedt, E. \\ Kristofer
}

Title: $\quad$ Effects of defects on the tensile strength of short-fibre composite materials

Year: $\quad 2014$

Version:

Please cite the original version:

Joffre, T., Miettinen, A., Wernersson, E. L., Isaksson, P., \& Gamstedt, E. K. (2014).

Effects of defects on the tensile strength of short-fibre composite materials.

Mechanics of Materials, 75(August), 125-134.

https://doi.org/10.1016/j.mechmat.2014.04.003

All material supplied via JYX is protected by copyright and other intellectual property rights, and duplication or sale of all or part of any of the repository collections is not permitted, except that material may be duplicated by you for your research use or educational purposes in electronic or print form. You must obtain permission for any other use. Electronic or print copies may not be offered, whether for sale or otherwise to anyone who is not an authorised user. 


\title{
Effects of defects on the tensile strength of short-fibre
}

\section{composite materials}

Thomas Joffre ${ }^{1}$, Arttu Miettinen ${ }^{2}$, Erik L.G. Wernersson ${ }^{3}$, Per Isaksson $^{1}$ and E. Kristofer Gamstedt $^{1 *}$

${ }^{1}$ Uppsala University, Ångström Laboratory, Department of Engineering Sciences, Box 534, SE-751 21, Uppsala, Sweden

${ }^{2}$ University of Jyväskylä, Department of Physics, Box 35, FI-40014, Finland

${ }^{3}$ Swedish University of Agricultural Sciences, Centre for Image Analysis, Box 337, SE-751 05 Uppsala, Sweden

*Corresponding author, e-mail address: kristofer.gamstedt@angstrom.uu.se

\begin{abstract}
Heterogeneous materials tend to fail at the weakest cross-section, where the presence of microstructural heterogeneities or defects controls the tensile strength. Short-fibre composites are an example of heterogeneous materials, where unwanted fibre agglomerates are likely to initiate tensile failure. In this study, the dimensions and orientation of fibre agglomerates have been analysed from three-dimensional images obtained by X-ray microtomography. The geometry of the specific agglomerate responsible for failure initiation has been identified and correlated with the strength. At the plane of fracture, a defect in the form of a large fibre agglomerate was almost inevitably found. These new experimental findings highlight a problem of some existing strength criteria, which are principally based on a rule of mixture of
\end{abstract}


the strengths of constituent phases, and not on the weakest link. Only a weak correlation was found between stress concentration induced by the critical agglomerate and the strength. A strong correlation was however found between the stress intensity and the strength, which underlines the importance of the size of largest defects in formulation of improved failure criteria for short-fibre composites. The increased use of three-dimensional imaging will facilitate the quantification of dimensions of the critical flaws.

Keywords: Strength, Agglomeration, X-ray microtomography, Composite materials

\section{Introduction}

The micromechanical models used to predict tensile strength of short-fibre composite materials, e.g. the Fukuda-Chou model (1982) or the Cox model (1952), have been inspired by micromechanical models for elastic properties, where the composite stiffness is determined from stiffness contributions of the constituents and effects of fibre orientation, fibre content and fibre length. These models may have surprisingly good predictive capabilities (Andersons and Joffe, 2011; Andersons et al., 2011; Virk et al., 2012) although mechanism-based criterion would be preferable, relying on the dimensions and properties of the most severe flaw initiating ultimate failure. With the advent and increased use of three-dimensional imaging techniques, most notably microfocus X-ray computed tomography, it is becoming easier to quantify these parameters (Buffière et al., 1999; Molnár and Bojtár, 2012). The present study is an attempt to investigate the correlation between composite strength and the physical features of the failure instigating flaw in an important class of brittle heterogeneous materials, namely short-fibre composites.

Although no previous studies have been found on the effects of critical flaws in the specific form of fibre agglomerates on strength, there are several studies on the relation between voids 
and strength among other mechanical properties of materials (Harper et al., 1987; Huang, 2004; Liu et al., 2006). The general consensus is that it is the most severe flaw or imperfection in the material that gives rise to brittle tensile failure. This is typically evidenced by fractography, where the examination of fracture surfaces by scanning electron microscopy can be traced back to the critical flaw that initiated crack growth leading to final failure (Greenhalgh, 1993; Hull, 1999). X-ray computed tomography (X $\mu C T)$ provides a useful nondestructive technique to quantify the microstructure of materials, not only on the fracture surface, but also in three-dimensions inside the material. To this end, $\mathrm{X} \mu \mathrm{CT}$ has already been used to measure crack growth and pores in concrete material under compressive loading (see e.g. Landis and Keane, 1999; Landis et al., 2003). For fibre composite materials, X $\mu$ CT has for instance been used to quantify internal damage (Schilling et al., 2005) and to observe crack opening due to tensile loading (Moffat et al., 2008).

In the present study, the samples were scanned before and after mechanical testing, allowing to identify the specific microstructural features that caused the failure. A short-fibre composite was chosen as model for a heterogeneous material, whose microstructure can be characterized by $\mathrm{X} \mu \mathrm{CT}$. Wood fibres were dispersed in a polylactide (PLA) matrix, forming a renewable composite material suitable for packaging applications. The cellulosic fibres tend to agglomerate in the injection-moulding process (Chinga-Carrasco et al., 2012), and thus form natural defects which could be related to the macroscopic strength.

In the following, the modelling approach is presented. Two strength models are outlined: (i) one accounting for the stress concentration factor caused by the largest detected flaw, (ii) one based on a fracture mechanics approach, where the size of the largest flaw is considered. Both of these models account the largest defect, in contrast to rule-of-mixture based criteria. The stress concentration factor approach accounts for the shape of the largest defect, and the stress intensity factor approach accounts also for the size of the largest defect. Thereafter, 
experimental details are presented, followed by the results and comparison of the correlation between the experimental results and the two models. Finally, some concluding remarks are given concerning developments of mechanism based models for strength predictions of heterogeneous composite materials.

\section{Stress analysis}

\subsection{Assumptions}

For the present model material, scanning electron microscopy revealed that the fibres were agglomerated into clusters of roughly ellipsoidal shape. Thus, three-dimensional general ellipsoids were considered versatile enough to describe the shape of the agglomerates for modelling purposes. Although it is a major idealization that the fibre agglomerates take an ellipsoidal shape with regard to the surrounding stress field, some hypothesis has to be made in order to make a quantitative analysis possible. We argument that this assumption is acceptable, since the aim at this stage is only to compare stress concentration effects with size-dependent stress-intensity effects on strength. Furthermore, the mechanical behaviour of the material is assumed to be elastic, since the macroscopic tensile behaviour was observed to be linearly elastic. Furthermore, the material was observed to fail in a brittle manner.

Fractographic studies of the material and other similar wood-fibre composites (ChingaCarrasco et al., 2010), have shown that fibre agglomerates easily debond from the surrounding thermoplastic matrix. Oksman et al. (2009) noticed that the strength of cellulose fibre composites was unaffected by the type of fibre when the fibre-matrix interface is poor, as is commonly the case for melt-processed cellulose fibre reinforced thermoplastics. Nyström et al. (2007) showed that the aspect ratio, i.e. the shape of the reinforcing units, had a large impact on composite strength, whereas the strength of the reinforcement has negligible influence on the measured strength for cellulose-fibre composites. Again, this was linked to 
poor adhesion and interfacial stress transfer. No residual resin was found on the fibre surfaces as would be expected in cohesive failure with a stronger interfacial bond. At the instance of failure, it is assumed that the largest agglomerate is completely debonded from the matrix, and that the agglomerate does not carry any load. Even if the fibre agglomerates would be bonded to the matrix, the stiffness of the fibre in the transverse direction is much inferior to typical polymer matrix systems (Bergander and Salmén, 2000; Joffre et al., 2014). If effects of lumen and other voids are taken into account, the transverse stiffness of the fibre agglomerates would be even lower.

Since the agglomerates tend to debond from the matrix and have relatively low transverse stiffness, they are regarded as ellipsoidal cavities. Thus the stiffness of the ellipsoidal inclusions is effectively set to zero. The dimensions, distributions, and orientation of defects inside the material have been characterized by $\mathrm{X} \mu \mathrm{CT}$. Since it is a non-destructive technique, the samples have been scanned before and after tensile testing. The three-dimensional images obtained have been used to identify the specific agglomerate responsible for the failure of the sample during mechanical loading.

The first step is to estimate the stress state around the ellipsoidal cavity surrounded by a resin rich region. Accurate analytical solutions exist for the stress state around an inclusion in an infinite matrix (Mura, 1987). The stress perturbation with regard to the far-field applied stress in the composite is different from the one caused by inclusion in the hypothetical infinite matrix. If we assume that the agglomerate is embedded in a matrix surrounded by the composite material, the strain continuity at the matrix/composite interface gives a relationship between the far-field applied stress in the composite case, $\sigma_{i j}^{\infty}$, and the fictitious applied stress in the infinite matrix case, $\sigma_{i j}^{0}$, 


$$
S_{i j k l}^{\mathrm{c}} \sigma_{k l}^{\infty}=S_{i j k l}^{\mathrm{m}} \sigma_{k l}^{0}
$$

where $S_{i j k l}^{\mathrm{c}}$ and $S_{i j k l}^{\mathrm{m}}$ are the compliance tensors of the composite and matrix, respectively. By multiplication with the elastic tensor for the isotropic matrix, $C_{i j k l}^{\mathrm{m}}$, a relation between the applied far-field stress and the effective stress applied on to the matrix embedded agglomerate is obtained:

$$
\sigma_{i j}^{0}=C_{i j k l}^{\mathrm{m}} S_{k l m n}^{\mathrm{c}} \sigma_{m n}^{\infty}
$$

For an aligned orthotropic composite and an isotropic matrix subjected to a uniaxial tensile stress, this equation can be simplified by neglecting the higher order terms and the relatively small off-axis stress components:

$$
\sigma_{z}^{0}=\frac{E^{\mathrm{m}}(1-v)}{E_{\mathrm{L}}^{\mathrm{c}}(1+v)(1-2 v)} \sigma_{z}^{\infty}
$$

where $E^{\mathrm{m}}$ and $v$ are the Young's modulus and Poisson's ratio of the matrix, respectively. $E_{\mathrm{L}}^{\mathrm{c}}$ is the Young's modulus of the composite in the longitudinal direction. For our composite material, and other orthotropic materials with the main material axis aligned along the load direction, the transverse stresses become negligible compared with the stress in the $z$ direction. This is why a uniaxial far-field stress state is assumed also for the modelling case with an ellipsoid surrounded by an isotropic matrix only.

In the following, the stress perturbation around agglomerates is determined in two different ways; (i) the stress concentration factor from Eshelby theory, and (ii) the stress intensity factor from fracture mechanics. The main difference by the two approaches is that the former accounts for the three-dimensional shape of the agglomerate, whereas the latter assumes that the defect is a two-dimension elliptical crack and thereby accounts for the length scale of the defect. In the latter case, the three-dimensional ellipsoidal inclusion is collapsed into to twodimensional elliptical crack in the direction of the most minor of the semi-principal axes. 
(a)

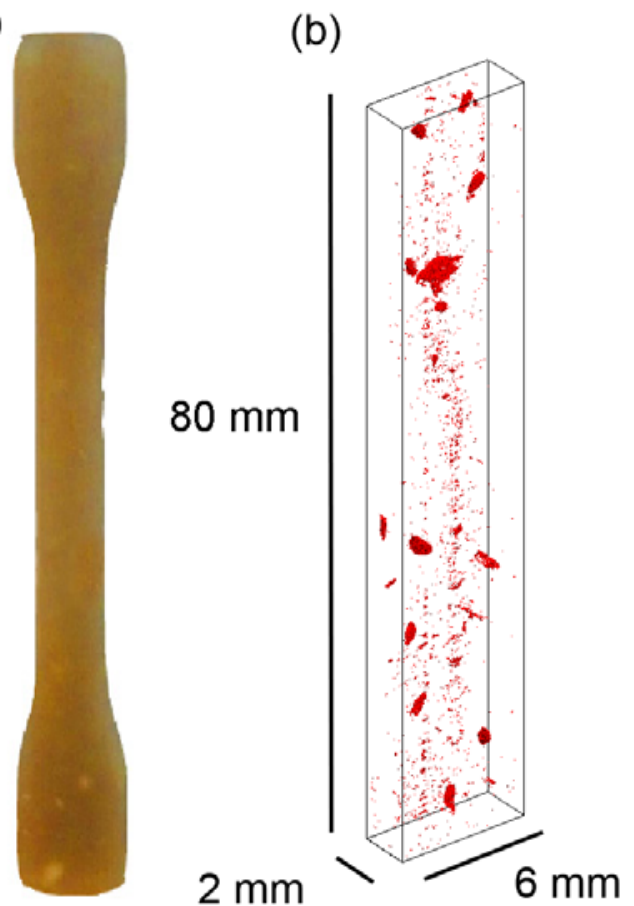

(c)

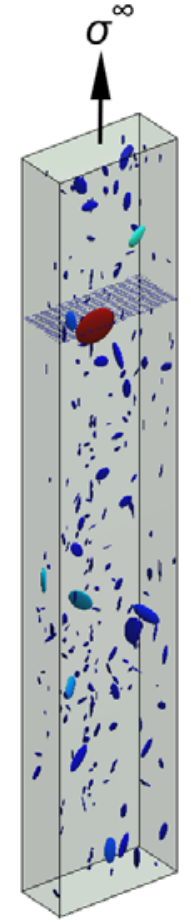

(d)

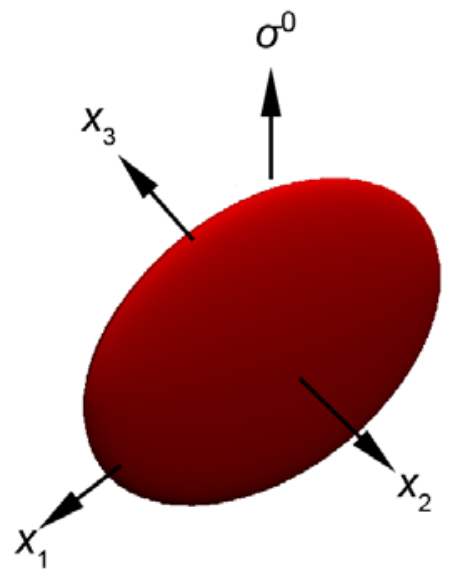

Figure 1. Identification of the critical agglomerate: (a) A dog bone composite sample. (b) Identification of the agglomerates with $\mathrm{X} \mu \mathrm{CT}$. (c) Geometrical modelling of the agglomerates using ellipsoids. The rupture plane is marked with small blue stripes. (d) Principal directions of the critical ellipsoid.

\subsection{Stress concentration factor}

The stress perturbation due to a general triaxial ellipsoidal cavity, such as the one represented in Fig. 2.a, was derived by Sadowsky and Sternberg (1949) using ellipsoidal coordinates and a harmonic function. A more straightforward method was then introduced by Eshelby (1957) who calculated the stress inside and outside the inhomogeneity using an equivalent inclusion method. The approach presented here is based on the work of Ju and Sun (1999, 2001), who presented an explicit solution for the stress field in the matrix using Eshelby's external tensor. 
The stress at a point $p=\left(x_{1}, x_{2}, x_{3}\right)$ in a Cartesian coordinate system, outside the ellipsoidal inhomogeneity centred at $(0,0,0)$, can be written as the sum of the far field stress in the infinite matrix, $\sigma_{i j}^{0}$, applied during mechanical testing and the stress perturbation due to the presence of the defect, $\sigma_{i j}^{\prime}(p)$

$$
\sigma_{i j}^{\mathrm{m}}(p)=\sigma_{i j}^{0}+\sigma_{i j}^{\prime}(p)
$$

Note that the stress field $\sigma_{i j}^{0}$ corresponds to the stress $\sigma_{z}^{0}$ expressed in the local coordinate system $\left(x_{1}, x_{2}, x_{3}\right)$ defined by the principal directions the ellipsoid. The stress perturbation in the matrix is expressed as the product of the exterior Eshelby tensor, $G_{i j k l}(p)$, and the eigentstrain $\varepsilon_{i j}^{0}$, expressed in the coordinate system defined by the directions of the axes of the ellipsoidal crack (Mura, 1987)

$$
\sigma_{i j}^{\prime}(p)=C_{i j k l}^{m} \cdot G_{k l m n}(p) \varepsilon_{m n}^{0}
$$

An explicit expression of the tensor $G_{i j k l}(p)$ is given in Gao and Ma (2010), and $\varepsilon_{i j}^{0}$ is given by Ju and Sun (1999, 2001). The full expressions of these two tensors can be found in Appendix A.
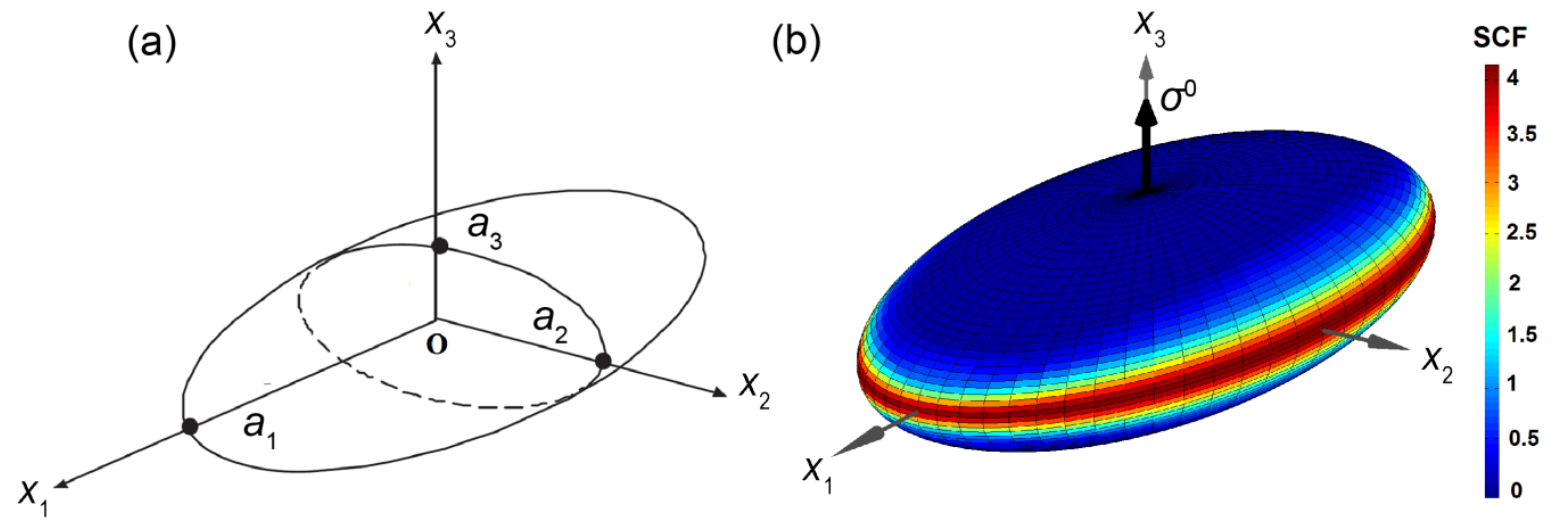

Figure 2. (a) Scheme of a general triaxial ellipsoidal cavity in a solid, and (b) the largest principal stress calculated imminently outside an ellipsoidal defect subjected to a stress, $\sigma^{0}$, aligned with the $x_{3}$ axis. 
To calculate the stress tensor outside the ellipsoid in a robust way, the method used by Healy (Healy, 2009) was advocated: using Eq. 2, the stress tensor in the matrix directly outside the ellipsoidal inclusion was calculated on the surface an ellipsoidal shell $0.01 \%$ larger than the inclusion. The stress concentration factor (SCF) is then given by the ratio between the largest first principal stress directly outside the ellipsoidal inclusion and the applied far-field stress, $\sigma_{i j}^{0}$ (see Fig. 2b). Note that it can be shown that the Eshelby exterior tensor, $G_{i j k l}(p)$, is size independent, i.e. it only depends on the ratio between the length of the principal axes of the ellipsoid $\left(a_{2} / a_{1}, a_{3} / a_{1}\right)$ (Healy, 2009). The SCF does thus not capture any length scale effect, i.e. the stress concentration is the same for a macroscopic agglomerate as for a microscopic one with the same shape and orientation. The strength of the material may be predicted by assuming that failure occurs when the criteria

$$
\sigma^{\text {th }}=\sigma^{\max } / \mathrm{SCF}
$$

is satisfied, where $\sigma^{\max }$ is the strength of a sample without any agglomerate. In the present study all samples had agglomerates of different geometries, and $\sigma^{\max }$ was identified by least square fitting of the experimental results.

\subsection{Stress intensity factor}

To account for size dependence, fracture mechanics can be used. However, the inhomogeneity has now to be considered as a flat two-dimensional crack. The assumption used here is that a crack will propagate in the plane perpendicular to the smallest of the semi-principal axes. Kassir and Sih (1966) have derived the stress intensity factors (SIFs) for an elliptic slanted crack. Contrary to the SCF, the SIFs depend on the size of the agglomerate. The SIFs for the fracture in opening and shearing mode are given by the expressions (Sih and Cha, 1974) 


$$
\begin{aligned}
& K_{1}=\frac{\sigma^{0} \sin ^{2} \beta}{E(k)} \sqrt{\frac{a_{2}}{a_{1}}}\left(a_{1}^{2} \sin ^{2} \alpha+a_{2}^{2} \cos ^{2} \alpha\right)^{1 / 4} \\
& K_{2} \\
& =-\frac{\sigma^{0} \sin \beta \cos \beta}{\left(a_{1}^{2} \sin ^{2} \alpha+a_{2}^{2} \cos ^{2} \alpha\right)^{1 / 4}}[1 \\
& \left.-\left(a_{2} / a_{1}\right)^{2}\right]\left(a_{2} / a_{1}\right)^{-1 / 2}\left[\frac{a_{1} \sin \alpha \sin \omega}{\left(k^{2}-v k^{\prime 2}\right) E(k)-v k^{\prime 2} K(k)}\right. \\
& \left.+\frac{a_{2} \cos \alpha \cos \omega}{\left(k^{2}-v\right) E(k)+k^{\prime 2} K(k)}\right]
\end{aligned}
$$

respectively, where $K_{1}$ and $K_{2}$ are the opening and shear mode stress intensity factors, $k=\left[1-\left(a_{2} / a_{1}\right)^{2}\right]^{1 / 2}$ and $k^{\prime}=1-k^{2}$. The quantities $E(k)$ and $K(k)$ correspond to the elliptical integrals of the first and second kind. The angles $\beta, \omega$ and $\alpha$ are defined in Fig. 3. The strength of the material may be predicted by assuming that the brittle failure occurs when the mixed-mode fracture criteria

$$
\left(\frac{K_{1}}{K_{1 \mathrm{c}}}\right)^{m}+\left(\frac{K_{2}}{K_{2 \mathrm{c}}}\right)^{n}=1
$$

is satisfied (Leonardo, 1979), where $K_{1 \mathrm{c}}$ and $K_{2 \mathrm{c}}$ are the fracture toughness values of the material in opening and shear mode, respectively. Here, $K_{1 \mathrm{c}}$ has been taken equal to 4 $\mathrm{MPa} \cdot \mathrm{m}^{1 / 2}$ according to experimentally determined values (Park et al., 2004) whereas $K_{2 \mathrm{c}}$ has been approximated using a ratio $K_{1 \mathrm{c}} / K_{2 \mathrm{c}}=0.8$ (Melin et al., 2000). Furthermore, the exponents $m$ and $n$ were taken to equal 1 and 2, respectively, following the experimental work of $\mathrm{Wu}(1967)$. 


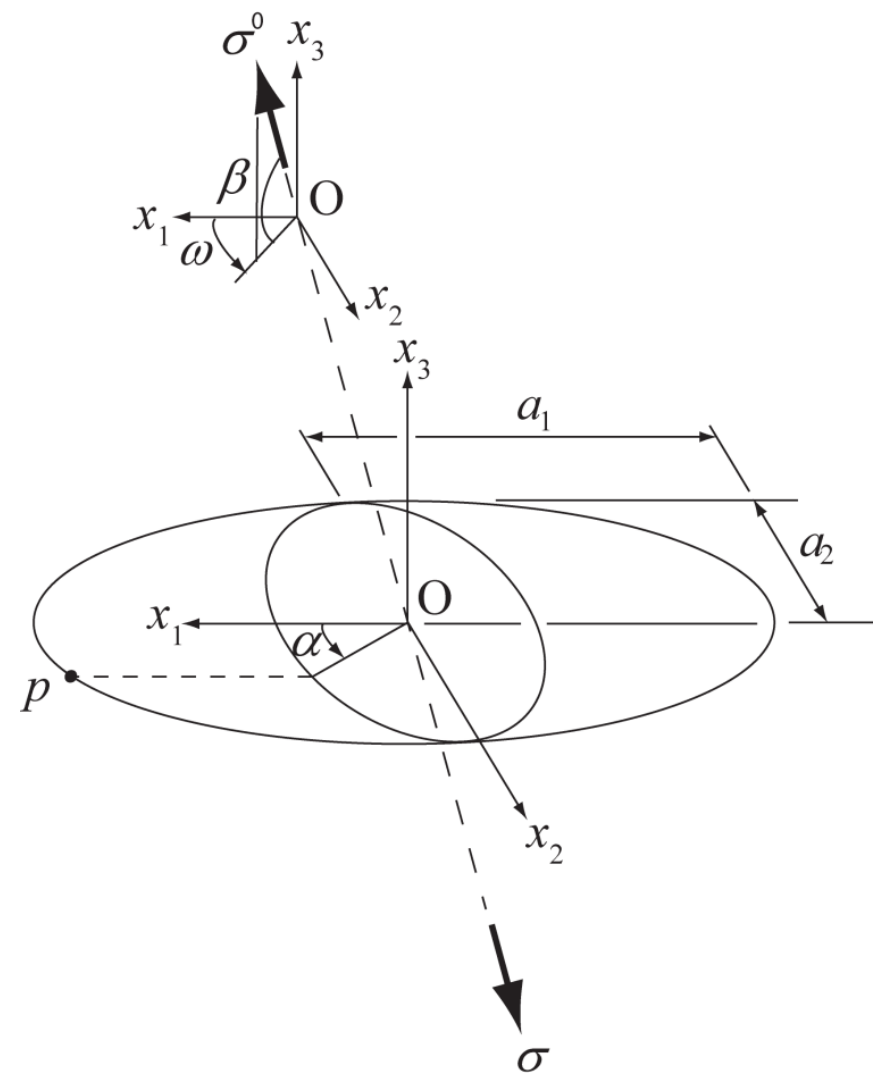

Figure 3. Elliptical crack subjected to inclined loading.

\section{Experimental procedures}

\subsection{Materials and manufacturing}

The fibres used in this study are pulp fibres from Norway spruce (Picea Abies). They were extracted from wood by three commonly used processes: chemo-thermomechanical pulping (CTMP), thermomechanical pulping (TMP) and kraft pulping. A description of the fibre separation processes can be found in Chinga-Carrasco et al. (2009).

The matrix material is polylactic acid (PLA). PLA is thermoplastic aliphatic polyester manufactured from starch-rich renewable resources such as maize, sugar beets or wheat. PLA 
has decent physical and mechanical properties, making it a candidate for substitution of certain petrochemical thermoplastics (Wolf et al., 2005). Due to the good adhesion to cellulose fibres, it is also suitable as matrix material in wood-fibre composites (Huda et al., 2006a; Huda et al., 2006b).

PLA and wood fibres were blended in a double-screw compounding unit at SINTEF Materials and Chemistry, Norway. The mixture obtained was then moulded to the form of dog-bone specimens. The mould was preheated in an oven for $10 \mathrm{~min}$ at $190^{\circ} \mathrm{C}$ before being compressed at $50^{\circ} \mathrm{C}$ and 10 bar during 10 seconds. The dimensions of the dog bones shaped specimens are $80 \mathrm{~mm} \times 6 \mathrm{~mm} \times 2 \mathrm{~mm}$ (Fig.1), according to the standard ASTM D638-IV. The weight fractions of fibres used in this study are $30 \%$ and $40 \%$. For each weight fraction ten samples were manufactured.

\subsection{X-ray micro-computed tomography}

$\mathrm{X} \mu \mathrm{CT}$ is a non-destructive method that is used to obtain the three-dimensional structure of a sample. Two-dimensional X-ray projection images of the area of interest are taken in multiple directions. The X-ray projections are used to computationally reconstruct the threedimensional microstructure of the sample, or more precisely, the special distribution of local X-ray attenuation coefficient. As the X-ray attenuation coefficient is different for fibres and matrix, the resulting images can be used to distinguish between the two phases (Kak and Slaney, 1988).

In this work the imaging was done using a SkyScan 1172 X-ray microtomograph employing X-ray energy of $40 \mathrm{keV}$ and power of $10 \mathrm{~W}$. Each sample was scanned before and after mechanical testing. The mechanical test itself was performed in a tensile machine, outside the chamber of the X- $\mu \mathrm{CT}$. The voxel size was 4.25 . The scanned area was chosen to contain the whole dog-bone except the wide clamping heads. In this way, it could be ensured that the 
mechanical failure of the sample would occur in a location inside the image, such that the geometry of the failure-initiating flaw could be analysed.

\subsection{Image analysis}

The reconstructed images were affected by a cupping artefact due to beam hardening which was removed using a post processing filter. The signal to noise ratio or contrast was low due to the similarity of X-ray absorption in fibres and matrix. Direct thresholding would not be very accurate and low pass filtering prior to thresholding would cause geometrical distortion (van Vliet, 1993). A maximum a posteriori classifier (Boykov and Funka-Lea, 2006; Greig et al., 1989), was used to overcome those problems. It was assumed that the pixel values corresponding to the two phases could be described by Gaussian distributions and that the clusters of interest were mostly homogeneous, i.e. not porous. As a final step, the classification into matrix, void or fibre was manually verified by inspecting the segmented slices side by side with the CT images.

An ellipsoid was then fitted to the voxels of each of the segmented agglomerates. The principal components of the points corresponding to the agglomerate were determined, and used as ellipsoid semi-axis directions (Psychol, 1901). The lengths of the semi-axes were standard deviations in the corresponding directions as given by the principal component analysis. The fitting of the ellipsoids is illustrated in Fig.1.

\subsection{Mechanical testing}

Prior to the test the specimens were kept at equilibrium conditions in an environmental controlled room where temperature was $23^{\circ} \mathrm{C}$ and the relative humidity $50 \%$ during seven days. The tensile tests were performed in a laboratory tensile test machine (Instron 5566). The 
machine was equipped with a $10 \mathrm{kN}$ load cell and a video extensometer. The specimens were tested at a cross-head speed of $1 \mathrm{~mm} / \mathrm{min}$.

\section{Result and discussion}

\subsection{Interface between fibre agglomerate and matrix}

Analysis of the $\mathrm{X} \mu \mathrm{CT}$ images confirmed the assumption that agglomerates have poor bonding with the PLA matrix, after manufacturing and after mechanical testing. For individual fibres or small agglomerates, the bonding was more complete. For the larger agglomerates, a large number of small voids can be observed at the agglomerate-matrix interface. Occasionally, the void had coalesced into larger interfacial openings. These interfacial voids appeared as black zones in the $\mathrm{X} \mu \mathrm{CT}$ images as shown in Fig. 4, since the X-ray absorption of the cavities was low.
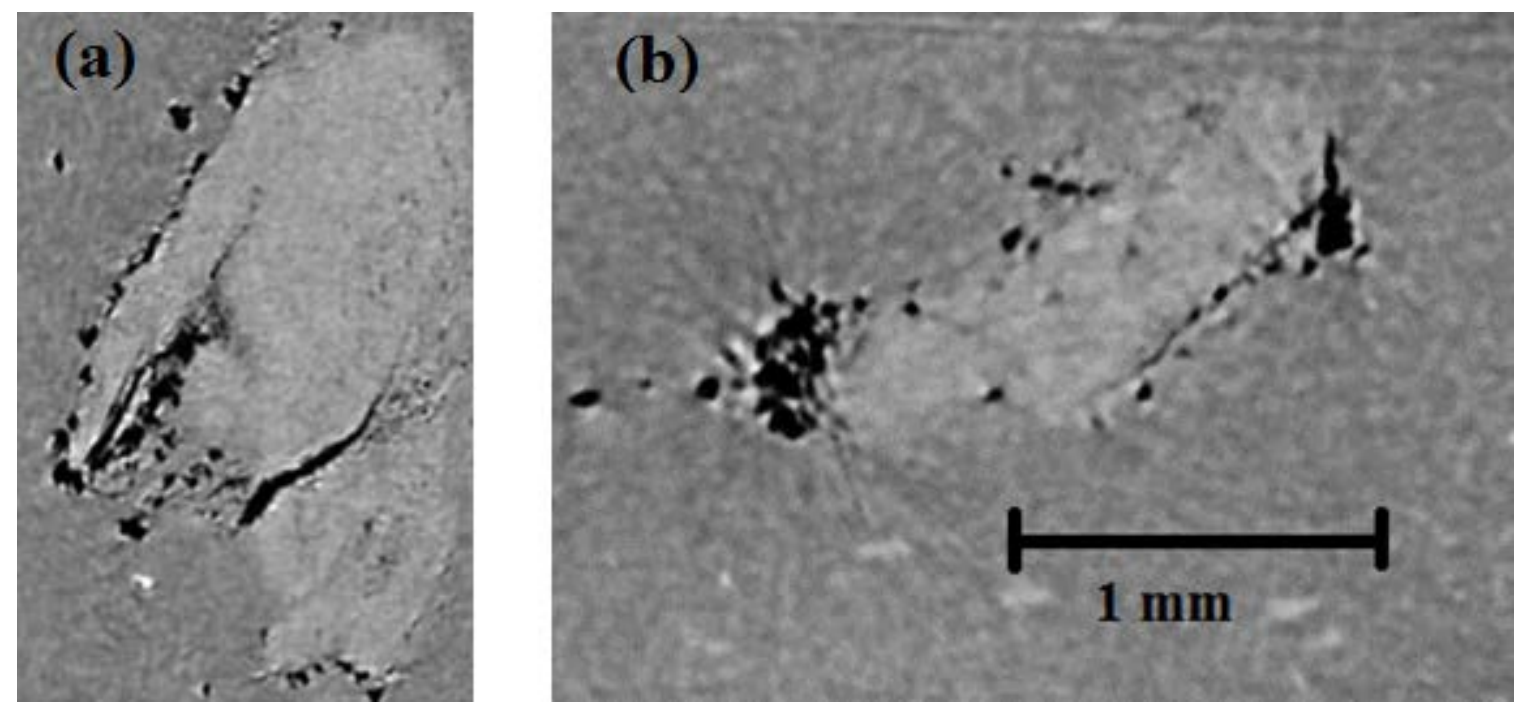

Figure 4. $\mathrm{X \mu CT}$ slices showing voids in the agglomerate-matrix interface for (a) kraft $30 \%$ material, and (b) kraft 40\% specimen. Unlike scanning electron micrographs of polished cross-sections, the $\mathrm{X \mu CT}$ images shows the interior microstructure and is generally not hampered by damage and artefacts from specimen machining and preparation. The length scale applied to both cross-section images. 
After mechanical testing, the presence of agglomerates at the fracture interface has been investigated. The $\mathrm{X} \mu \mathrm{CT}$ scans consistently confirmed the presence of large agglomerates at the fracture surface. An example with extremely large fibre agglomerate is presented in Fig. 5: the upper part of the fracture surface is convex whereas the lower part is concave. From this unevenness of either fracture surface, there are many fibres protruding, which reveals the presence the fibre agglomerate. Fracture has likely initiated from the exposed interface between the agglomerate and the surrounding matrix.
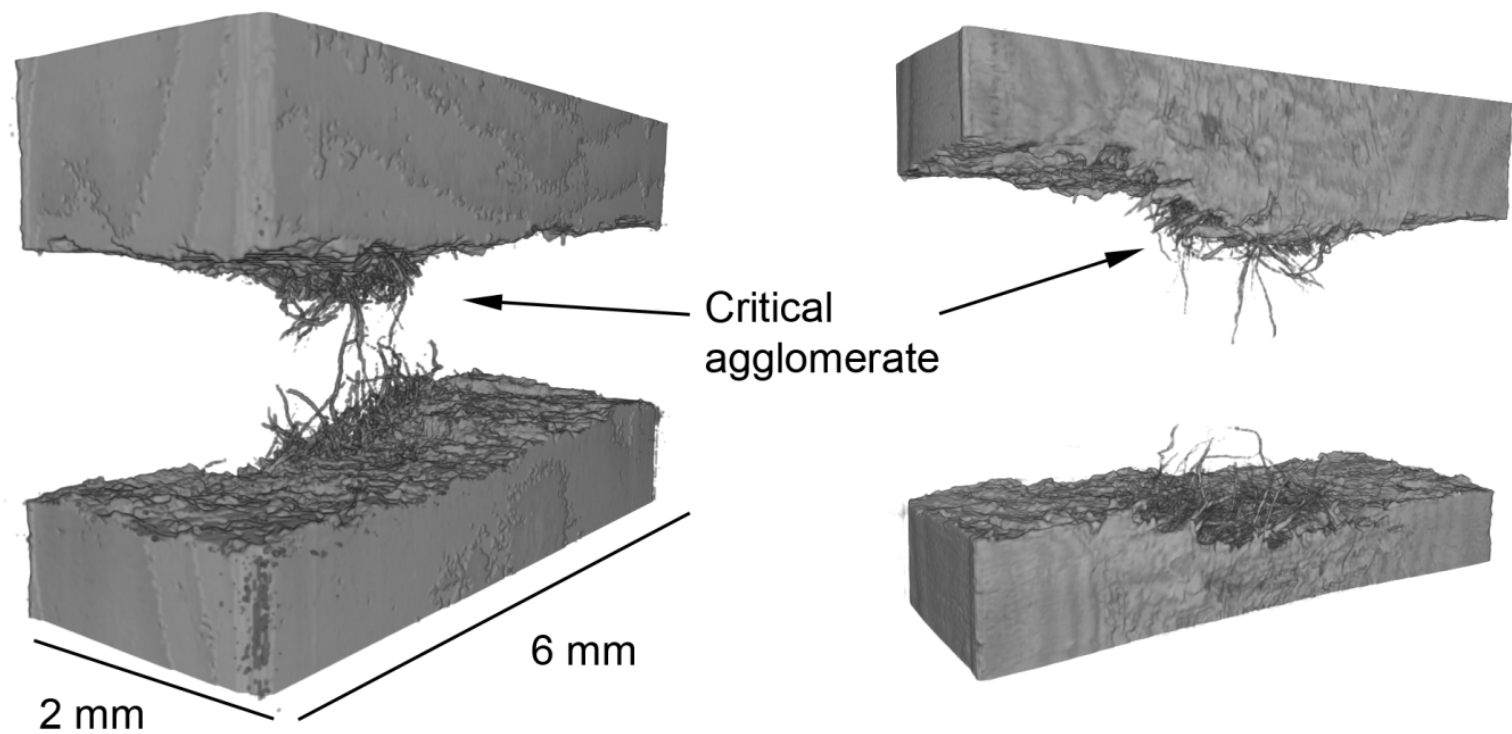

Figure 5. Visualisations of a $\mathrm{X} \mu \mathrm{CT}$ image of the fracture surface of a sample $(30 \% \mathrm{kraft}$ fibres). The arrows are pointing toward the critical agglomerate, which initiated final failure.

\subsection{Stress field and model validation}

The analytical solution for the stress concentration presented in the modelling section above and in Appendix A has been validated by comparing the analytical results with the ones obtained using a commercial finite element (FE) software, ANSYS 11. Symmetry planes were used when applicable to reduce the computational time. Around 50000 tetrahedral elements 
were created for each simulation. The relative difference between the SCF predictions using the analytical model and the one obtained through the FE simulation did not exceed 5\% in any of cases, as compiled in Table 1. An example of this comparison is illustrated in Fig. 6.

(a)

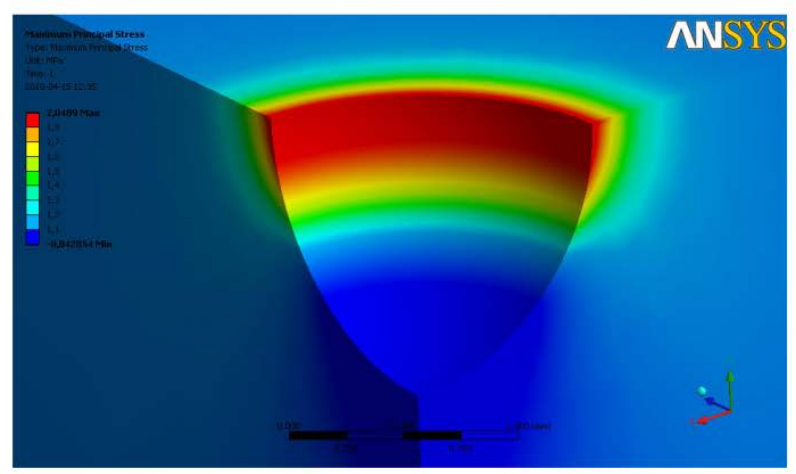

(b)

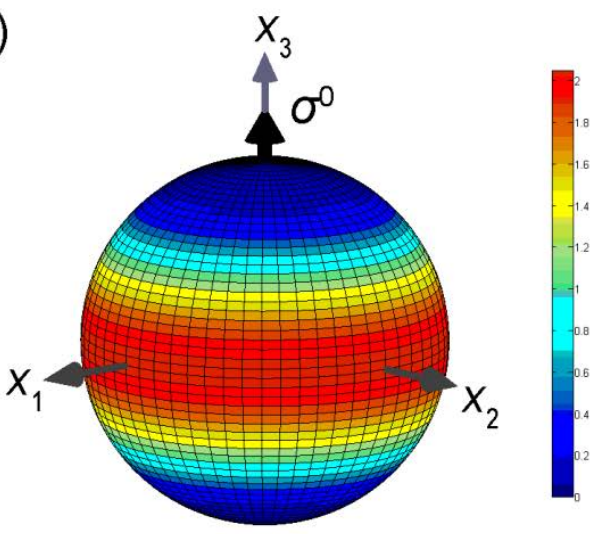

Figure 6. Stress concentration around a sphere subjected to traction determined by (a) FE simulation visualized in a matrix octant, and (b) by the analytical solution, shown on the surface of the inclusion. In both cases the SCF is equal to 2.05 for the chosen material properties.

Table 1 Validation of the analytical solution of the stress concentration by comparison with the results from the finite element analysis.

\begin{tabular}{|c|c|c|c|c|}
\hline $\begin{array}{l}\text { Inclusion } \\
\left(a_{1}, a_{2}, a_{3}\right)\end{array}$ & $\begin{array}{l}\text { SCF: Analytica } \\
\text { solution }\end{array}$ & $\begin{array}{l}\text { SCF: } \\
\text { simulation }\end{array}$ & FE & Difference (\%) \\
\hline Sphere $(1,1,1)$ & 2.05 & 2.05 & & -0.2 \\
\hline $\begin{array}{ll}\text { Prolate } & \text { Straight } \\
(1,1,5) & \end{array}$ & 1.13 & 1.12 & & 1.0 \\
\hline $\begin{array}{l}\text { Oblate Straight } \\
(1,5,5)\end{array}$ & 7.14 & 7.32 & & -2.5 \\
\hline Prolate, tilted $60^{\circ}$ & 2.45 & 2.58 & & -5.0 \\
\hline Oblate, tilted $60^{\circ}$ & 3.42 & 3.36 & & 1.7 \\
\hline
\end{tabular}

\subsection{Strength correlation}


The material properties used to predict the strength are summarized in Table 2. $\boldsymbol{E}^{\mathbf{m}}$ and $\boldsymbol{v}$ were set to $2.3 \mathrm{GPa}$ and 0.35 respectively according to literature data (Almgren et al., 2008; Almgren et al., 2010). The Young's modulus, $\boldsymbol{E}_{\mathbf{L}}^{\mathbf{c}}$ can be estimated using for instance the laminate analogy (Joffre et al., 2013) but since tensile tests were carried out within this study, the experimental value were used. The results obtain by using the SIF and the SCF approaches are presented in Fig. 7. Additional information about the value of size and orientation of the largest ellipsoid for the different specimens is provided in Appendix B.

The results obtained by using the SCF approach are presented in Fig. 7(a). The predicted strength using the SCF theory does not correlate well with the experimental results. The coefficient of determination is merely $R^{2}=0.22$. The results obtained by using the SIF approach are presented in Fig. 7(b). In contrast to the SCF predictions, there is a clear correlation between the strength values obtained from experimental testing and from the length-scale depended SIF model. The coefficient of determination is $R^{2}=0.67$, which shows an apparent correlation despite the large scatter in strength. The measured strength of the 9 samples having a weight fraction of $30 \%$ ranged from 55 to $70 \mathrm{MPa}$, whereas the strength of the 7 samples with a weight fraction of $40 \%$ composite ranged from 61 to $74 \mathrm{MPa}$. Existing models used for the prediction of the strength of short fibre composite, (Cox, 1952; Fu and Lauke, 1996; Fukuda and Chou, 1981, 1982; Zhu et al., 1994), do not account for the variability of strength. With a criterion not only based on the overall properties of the constituents, but also including the dimensions of the critical defect, it is possible to reduce the scatter and improve the predictive precision. The most straight forward way to account for the size of a critical effect is to adopt a fracture mechanics approach, as has been advocated in the presented study. Since the defects are generally inclined, it was necessary to quantify fracture toughness for the different modes of fracture, and use a mixed-mode fracture criterion. 


\section{Table 2}

Average measured longitudinal Young's modulus and co-fitted strength of a virtual composite having no agglomerate (Eq. 6).

\begin{tabular}{lll}
\hline$w_{\mathrm{f}}(\%)$ & $E_{L}^{\mathrm{c}}(\mathrm{GPa})$ & $\sigma^{\mathrm{max}}(\mathrm{MPa})$ \\
\hline 30 & 5.9 & 90 \\
\hline 40 & 6.5 & 96 \\
\hline
\end{tabular}

(a)

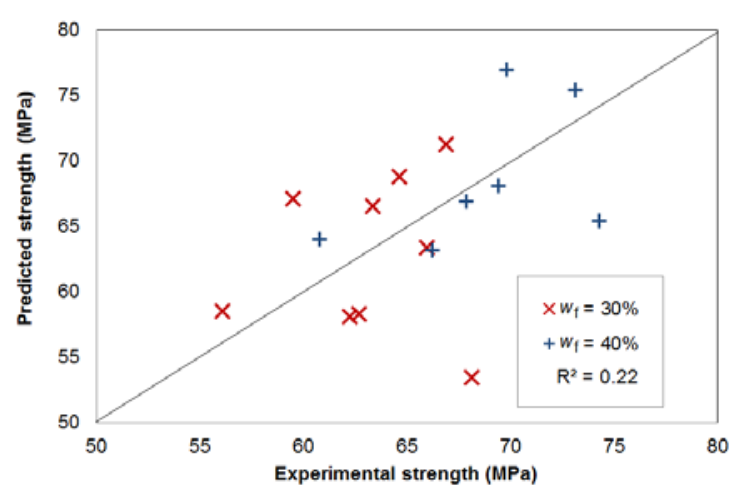

(b)

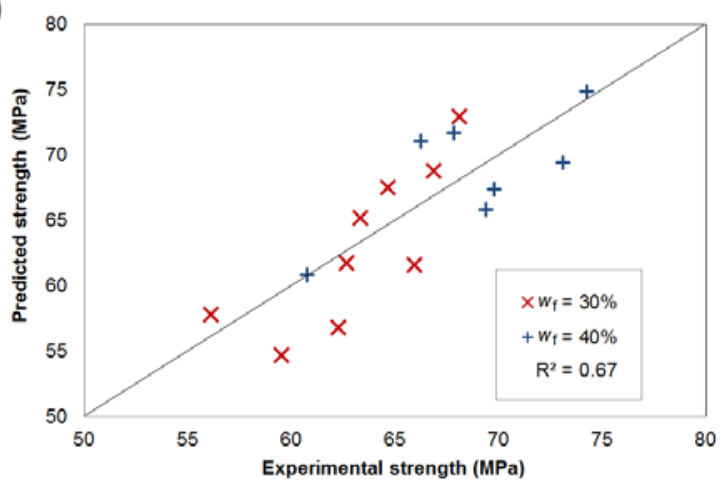

Figure 7. Strength prediction using (a) the stress concentration factor (b) the stress intensity factors and a mixed-mode fracture criterion.

The bottle-neck has hitherto been to measure the geometry of the critical defect. With the ongoing development of X-ray computed tomography, this hurdle is becoming increasingly conquerable, which opens doors to development of more accurate and mechanism-based failure criteria. The simple linear-elastic fracture mechanics model used here to relate the strength to the size of the critical defect could be refined to reduce scatter and improve predictability further. The first assumption here is that the agglomerates are effectively acting as voids, i.e. they are completely debonded from the matrix, as observed experimentally at high applied stresses close to failure. The interaction between adjacent fibre agglomerates is also neglected for composites with a low volume fraction of fibres. Some shielding effect can be expected, though, by the presence of other smaller agglomerates in the vicinity of the critical defect. Furthermore, the agglomerates are not perfectly ellipsoidal, but have three- 
dimensional irregular features. In average, however, the agglomerates can be regarded as flat ellipsoids, not far from two-dimensional ellipsoidal cracks. The suppression of the minor radius is motivated by the fact that we can directly investigate the effect of agglomerate size on strength by a fracture mechanics model.

\section{Conclusions}

Failure criteria for strength of short fibre composites are generally inspired by success of various rules of mixtures for homogenization of elastic properties. This experimental study shows that failure of wood-fibre reinforced PLA composite is initiated from the most severe defect in the material, typically a large transversely oriented fibre agglomerate. From X-ray computed tomography, it was possible to measure the dimensions and orientation of the critical defect. The defect was modelled by an ellipsoid, but it was found that the stress concentration caused by the ellipsoidal inclusion did not correlate very well with the measured tensile strength. This led to the hypothesis that not only the shape of the critical defect, but also the length scale of the defect affects the strength. A significantly better correlation with the measured strength was obtained by use of a mixed-mode fracture criterion. It was thus demonstrated with three-dimensional image analysis that the strength of a heterogeneous composite material is controlled by the geometry and size of its most severe defect, and not only by average mechanical properties of the composite constituents. Further work is, however, necessary to formulate an improved strength model for engineering purposes. X-ray computed tomography techniques also need to be rationalized to identify and quantify critical interior defects.

\section{Acknowledgements}


This work was financially supported by WoodWisdom-Net, project WoodFibre3D. Former colleagues at Wallenberg Wood Science Centre and Department of Solid Mechanics at KTH are gratefully acknowledged for their help in tensile testing and numerical analysis.

\section{Appendix A. Stress concentration factor}

For a point $p=\left(x_{1}, x_{2}, x_{3}\right)$ outside the ellipsoidal inclusion centred at $O=(0,0,0)$. The Eshelby tensor, used in Eq. 1 can be expressed as (Gao and Ma, 2010)

$$
\begin{aligned}
\mathrm{G}_{i j l m}(p)=S_{i l}^{(1)} & \delta_{i j} \delta_{l m}+S_{i j}^{(2)}\left(\delta_{i l} \delta_{j m}+\delta_{i m} \delta_{j l}\right) \\
& +S_{i}^{(3)} \omega_{l} \omega_{m} \delta_{i j} x_{l} x_{m}+S_{l}^{(4)} \omega_{i} \omega_{j} \delta_{l m} x_{l} x_{j} \\
& +S_{i}^{(5)}\left(\omega_{j} \omega_{m} \delta_{i l} x_{j} x_{m}+\omega_{j} \omega_{l} \delta_{i m} x_{j} x_{l}\right) \\
& +S_{j}^{(6)}\left(\omega_{j} \omega_{m} \delta_{j l} x_{i} x_{m}+\omega_{i} \omega_{m} \delta_{j m} x_{i} x_{l}\right) \\
& +S_{i j l m}^{(7)} x_{i} x_{j} x_{l} x_{m}
\end{aligned}
$$

where

$$
\begin{gathered}
S_{i l}^{(1)}(\lambda)=\frac{1}{8 \pi(1-v)}\left[2 v I_{i}(\lambda)-I_{l}(\lambda)+a_{i}^{2} I_{i l}(\lambda)\right] \\
S_{i j}^{(2)}(\lambda)=\frac{1}{8 \pi(1-v)}\left\{(1-v)\left[I_{i}(\lambda)+I_{j}(\lambda)\right]-I_{j}(\lambda)+a_{i}^{2} I_{i l}(\lambda)\right\} \\
S_{i}^{(3)}(\lambda)=\frac{a_{1} a_{2} a_{3}}{\Delta Z} \frac{\lambda}{2(1-v)} \omega_{i} \\
S_{l}^{(4)}(\lambda)=\frac{a_{1} a_{2} a_{3}}{\Delta Z} \frac{1}{2(1-v)}\left(\lambda \omega_{l}-2 v\right) \\
S_{I}^{(5)}(\lambda)=\frac{a_{1} a_{2} a_{3}}{\Delta Z} \frac{1}{2(1-v)}\left(\lambda \omega_{i}-1+v\right) \\
S_{J}^{(6)}(\lambda)=\frac{a_{1} a_{2} a_{3}}{\Delta Z} \frac{1}{2(1-v)}\left(\lambda \omega_{j}-1+v\right) \\
S_{i j l m}^{(7)}(\lambda)=\frac{a_{1} a_{2} a_{3}}{\Delta Z^{2}} \frac{1}{2(1-v)} \omega_{i} \omega_{j} \omega_{l} \omega_{m}\left[2-2 \lambda\left(\omega_{i}+\omega_{j}+\omega_{l}+\omega_{m}\right)-\lambda \theta+\frac{4 \lambda \eta}{Z}\right]
\end{gathered}
$$

and 


$$
\omega_{i}=\frac{1}{a_{i}^{2}+\lambda}
$$

where $v$ is the Poisson ratio. The constant $\lambda$ is determined by a new imaginary ellipsoid for each point $p$ such as

$$
\frac{x_{1}^{2}}{a_{1}^{2}+\lambda}+\frac{x_{2}^{2}}{a_{2}^{2}+\lambda}+\frac{x_{3}^{2}}{a_{3}^{2}+\lambda}=1
$$

Since Eq. A4 does not have any simple analytical solution, $\lambda$ has been evaluated numerically. Note that since the point $p$ is outside the inclusion $\lambda$ is strictly positive. The parameters $\Delta, Z, \theta$ and $\eta$ are determined by the relations

$$
\begin{gathered}
\Delta=\sqrt{\left(a_{1}^{2}+\lambda\right)\left(a_{2}^{2}+\lambda\right)\left(a_{3}^{2}+\lambda\right)}, \quad Z=\frac{x_{m}^{2}}{\left(a_{m}^{2}+\lambda\right)^{2}}, \\
\theta=\omega_{m} \text { and } \eta=\frac{x_{m}^{2}}{\left(a_{m}^{2}+\lambda\right)^{3}} .
\end{gathered}
$$

The functions $I_{i}(\lambda)$ and $I_{i j}(\lambda)$ in Eq. A2 are defined by the integrals

$$
\begin{aligned}
& I_{i}(\lambda)=2 \pi a_{1} a_{2} a_{3} \int_{\lambda}^{\infty} \frac{\mathrm{d} t}{\left(a_{i}^{2}+t\right) \sqrt{\left(a_{1}^{2}+t\right)\left(a_{2}^{2}+t\right)\left(a_{3}^{2}+t\right)}} \\
& I_{i j}(\lambda) \\
& =2 \pi a_{1} a_{2} a_{3} \int_{\lambda}^{\infty} \frac{\mathrm{d} t}{\left(a_{i}^{2}+t\right)\left(a_{j}^{2}+t\right) \sqrt{\left(a_{1}^{2}+t\right)\left(a_{2}^{2}+t\right)\left(a_{3}^{2}+t\right)}}
\end{aligned}
$$

In the case where the inclusion is a void, the eigenstrain $\varepsilon^{0}$ in Eq. 1 can be expressed as

$$
\varepsilon_{i j}^{0}=-\left(\tilde{S}_{i j k l}-I_{i j k l}\right)^{-1} \varepsilon_{k l}^{\infty}
$$

Where $I_{i j k l}$ is the identity tensor, $\varepsilon_{i j}^{\infty}$ corresponds to the strain due to the applied far-field stress $\left(\sigma_{i j}^{\infty}=C_{i j k l}^{m} \varepsilon_{i j}^{\infty}\right)$, and $\tilde{S}$ is Eshelby interior tensor defined as (Eshelby, 1957) 


$$
\tilde{S}_{i j k l}=\frac{1}{4(1-v)}\left[S_{i k}^{(1)}(0) \delta_{i j} \delta_{k l}+S_{i j}^{(2)}(0)\left(\delta_{i k} \delta_{j l}+\delta_{i l} \delta_{j k}\right)\right]
$$

where $S_{i k}^{(1)}$ and $S_{i j}^{(2)}$ can be derived from Eq. A2 by setting $\lambda$ equal to 0 .

\section{Appendix B. Geometry of the largest agglomerates for the tensile-tested specimens}

\section{Table B1}

Weight fraction of fibres, ultimate tensile strength, principal semi-axis lengths and main inclination angle of the critical defect for each sample.

\begin{tabular}{llllll}
\hline$w_{\mathrm{f}}[\%]$ & $\sigma_{\max }[\mathrm{MPa}]$ & $a_{1}[\mu \mathrm{m}]$ & $a_{2}[\mu \mathrm{m}]$ & $a_{3}[\mu \mathrm{m}]$ & $\beta\left[^{\circ}\right]$ \\
\hline 30 & 62.3 & 357 & 183 & 96 & 71 \\
& 62.7 & 177 & 119 & 44 & 71 \\
& 68.1 & 116 & 73 & 28 & 75 \\
& 56.1 & 707 & 187 & 124 & 76 \\
& 59.5 & 234 & 100 & 58 & 71 \\
& 66.9 & 169 & 119 & 46 & 44 \\
& 63.4 & 209 & 119 & 47 & 59 \\
& 64.7 & 125 & 114 & 27 & 56 \\
& 66.0 & 163 & 119 & 42 & 80 \\
\hline 40 & 67.9 & 218 & 117 & 61 & 65 \\
& 69.4 & 344 & 161 & 94 & 85 \\
& 73.1 & 473 & 246 & 88 & 88 \\
& 60.8 & 147 & 126 & 47 & 60 \\
& 66.2 & 257 & 201 & 72 & 66 \\
& 69.8 & 132 & 109 & 30 & 69 \\
& 74.3 & 158 & 131 & 51 & 74 \\
\hline
\end{tabular}

\section{References}

Almgren, K.M., Åkerholm, M., Gamstedt, E.K., Salmen, L., Lindström, M., 2008. Effects of Moisture on Dynamic Mechanical Properties of Wood Fiber Composites Studied by Dynamic FT-IR Spectroscopy. Journal of Reinforced Plastics and Composites 27, 1709-1721.

Almgren, K.M., Gamstedt, E.K., Varna, J., 2010. Contribution of wood fiber hygroexpansion to moisture induced thickness swelling of composite plates. Polymer Composites 31, 762-771. Andersons, J., Joffe, R., 2011. Estimation of the tensile strength of an oriented flax fiberreinforced polymer composite. Composites Part A 42, 1229-1235.

Andersons, J., Joffe, R., Spārniņš, E., Weichert, D., 2011. Modeling the effect of reinforcement discontinuity on the tensile strength of UD flax fiber composites. Journal of Materials Science, 1-7. 
Bergander, A., Salmén, L., 2000. Variations in transverse fibre wall properties: relations between elastic properties and structure. Holzforschung 54, 654-660.

Boykov, Y., Funka-Lea, G., 2006. Graph cuts and efficient ND image segmentation. International Journal of Computer Vision 70, 109-131.

Buffière, J.-Y., Maire, E., Cloetens, P., Lormand, G., Fougères, R., 1999. Characterization of internal damage in a MMC using X-ray synchrotron phase contrast microtomography. Acta Materialia 47, 1613-1625.

Chinga-Carrasco, G., Johnsen, P.O., Oyaas, K., 2010. Structural quantification of wood fibre surfaces--Morphological effects of pulping and enzymatic treatment. Micron 41, 648-659.

Chinga-Carrasco, G., Lenes, M., Johnsen, P., Hult, E.L., 2009. Computer-assisted scanning electron microscopy of wood pulp fibres: Dimensions and spatial distributions in a polypropylene composite. Micron 40, 761-768.

Chinga-Carrasco, G., Miettinen, A., Hendriks, C.L.L., Gamstedt, E.K., Kataja, M., 2012. Structural Characterisation of Kraft Pulp Fibres and Their Nanofibrillated Materials for Biodegradable Composite Applications. InTech.

Cox, H., 1952. The elasticity and strength of paper and other fibrous materials. British Journal of Applied Physics 3, 72-79.

Eshelby, J.D., 1957. The determination of the elastic field of an ellipsoidal inclusion, and related problems. Proceedings of the Royal Society of London. Series A. 241, 376.

Fu, S.Y., Lauke, B., 1996. Effects of fiber length and fiber orientation distributions on the tensile strength of short-fiber-reinforced polymers. Composites Science and Technology 56, 1179-1190.

Fukuda, H., Chou, T.W., 1981. A probabilistic theory for the strength of short fibre composites. Journal of Materials Science 16, 1088-1096.

Fukuda, H., Chou, T.W., 1982. A probabilistic theory of the strength of short-fibre composites with variable fibre length and orientation. Journal of Materials Science 17, 1003-1011.

Gao, X.L., Ma, H., 2010. Strain gradient solution for Eshelby's ellipsoidal inclusion problem. Proceedings of the Royal Society A: Mathematical, Physical and Engineering Science 466, 2425-2446.

Greenhalgh, E., 1993. Delamination growth in carbon-fibre composite structures. Composite Structures 23, 165-175.

Greig, D., Porteous, B., Seheult, A.H., 1989. Exact maximum a posteriori estimation for binary images. Journal of the Royal Statistical Society. Series B, 271-279.

Harper, B., Staab, G., Chen, R., 1987. A note on the effects of voids upon the hygral and mechanical properties of AS4/3502 graphite/epoxy. Journal of composite materials 21, 280.

Healy, D., 2009. Short note: Elastic field in 3D due to a spheroidal inclusion-MATLAB TM code for Eshelby's solution. Computers \& Geosciences 35, 2170-2173.

Huang, Y.Y., 2004. The Effect of Nanotube Waviness and Agglomeration on the Elastic Property of Carbon Nanotube-Reinforced Composites. Journal of Engineering Materials and Technology 126, 250-257.

Huda, M., Drzal, L., Misra, M., Mohanty, A., 2006a. Wood fiber reinforced poly (lactic acid) composites: Evaluation of the physicomechanical and morphological properties. Journal of Applied Polymer Science 102, 4856-4869.

Huda, M.S., Drzal, L.T., Mohanty, A.K., Misra, M., 2006b. Chopped glass and recycled newspaper as reinforcement fibers in injection molded poly (lactic acid)(PLA) composites: a comparative study. Composites Science and Technology 66, 1813-1824.

Hull, D., 1999. Fractography: observing, measuring and interpreting fracture surface topography. Cambridge University Press. 
Joffre, T., Neagu, R.C., Bardage, S.L., Gamstedt, E.K., 2014. Modelling of the hygroelastic behaviour of normal and compression wood tracheids. Journal of structural biology 185, 8998.

Joffre, T., Wernersson, E.L., Miettinen, A., Luengo Hendriks, C.L., Gamstedt, E.K., 2013. Swelling of cellulose fibres in composite materials: constraint effects of the surrounding matrix. Composites Science and Technology 74, 52-59.

Ju, J., Sun, L., 1999. A novel formulation for the exterior-point Eshelby's tensor of an ellipsoidal inclusion. Journal of Applied Mechanics 66, 570.

Ju, J., Sun, L., 2001. Effective elastoplastic behavior of metal matrix composites containing randomly located aligned spheroidal inhomogeneities. Part I: micromechanics-based formulation. International Journal of Solids and Structures 38, 183-201.

Kak, A., Slaney, M., 1988. Principles of computerized tomographic imaging IEEE Press. New York.

Kassir, M.K., Sih, G.C., 1966. Three-dimensional stress distribution around an elliptical crack under arbitrary loadings. Journal of Applied Mechanics 33, 601.

Landis, E.N., Keane, D.T., 1999. X-ray microtomography for fracture studies in cement-based materials. SPIE's International Symposium on Optical Science, Engineering, and Instrumentation, 105-113.

Landis, E.N., Nagy, E.N., Keane, D.T., 2003. Microstructure and fracture in three dimensions. Engineering Fracture Mechanics 70, 911-925.

Leonardo, G., 1979. Fracture toughness characterization of materials under multiaxial loading. International Journal of Fracture 15, 537-552.

Liu, L., Zhang, B.M., Wang, D.F., Wu, Z.J., 2006. Effects of cure cycles on void content and mechanical properties of composite laminates. Composite Structures 73, 303-309.

Melin, L.G., Neumeister, J.M., Pettersson, K.B., Johansson, H., Asp, L.E., 2000. Evaluation of four composite shear test methods by digital speckle strain mapping and fractographic analysis. Journal of Composites Technology \& Research 22, 161-172.

Moffat, A., Wright, P., Buffière, J.-Y., Sinclair, I., Spearing, S.M., 2008. Micromechanisms of damage in $0^{\circ}$ splits in a [90/0]s composite material using synchrotron radiation computed tomography. Scripta materialia 59, 1043-1046.

Molnár, G., Bojtár, I., 2012. Effects of manufacturing inhomogeneities on strength properties of float glass. Mechanics of Materials 59, 1-13.

Mura, T., 1987. Micromechanics of defects in solids. Springer.

Nyström, B., Joffe, R., Långström, R., 2007. Microstructure and strength of injection molded natural fiber composites. Journal of Reinforced Plastics and Composites 26, 579-599.

Oksman, K., Mathew, A.P., Långström, R., Nyström, B., Joseph, K., 2009. The influence of fibre microstructure on fibre breakage and mechanical properties of natural fibre reinforced polypropylene. Composites Science and Technology 69, 1847-1853.

Park, S.D., Todo, M., Arakawa, K., 2004. Effect of annealing on the fracture toughness of poly (lactic acid). Journal of Materials Science 39, 1113-1116.

Psychol, J.E., 1901. 1. Pearson K: On lines and planes of closest fit to systems of points in space. Phil Mag 6, 559-572.

Sadowsky, M.A., Sternberg, E., 1949. Stress concentration around a triaxial ellipsoidal cavity. Journal of Applied Mechanics 16, 149-157.

Schilling, P.J., Karedla, B.R., Tatiparthi, A.K., Verges, M.A., Herrington, P.D., 2005. X-ray computed microtomography of internal damage in fiber reinforced polymer matrix composites. Composites Science and Technology 65, 2071-2078.

Sih, G., Cha, B., 1974. A fracture criterion for three-dimensional crack problems. Engineering Fracture Mechanics 6, 699-723. 
van Vliet, L.J., 1993. Grey-Scale Measurements in Multi-Dimensional Digitized Images. Delft University of Technology, Delft pp. 1-259.

Virk, A., Hall, W., Summerscales, J., 2012. Modulus and strength prediction for natural fibre composites. Materials Science and Technology 28, 864-871.

Wolf, O., Crank, M., Patel, M., Marscheider-Weidemann, F., Schleich, J., Hüsing, B., Angerer, G., 2005. Techno-economic feasibility of large-scale production of bio-based polymers in Europe.

Wu, E.M., 1967. Application of fracture mechanics to anisotropic plates. Journal of Applied Mechanics 34(4), 967-974.

Zhu, Y., Zong, G., Manthiram, A., Eliezer, Z., 1994. Strength analysis of random short-fibrereinforced metal matrix composite materials. Journal of Materials Science 29, 6281-6286. 